\title{
Propuestas para el acopio de datos y el análisis de las competencias en el currículum universitario
}

\author{
Proposals for data collection and analysis of competencies in the university curriculum
}

\author{
Ana Esther Escalante ${ }^{a}$,Evelyn E. Moctezuma-Ramírez ${ }^{b}$, Fran J. García-García ${ }^{c}$
}

\begin{abstract}
:
The objective of this article is to offer alternatives to a series of methodological concerns for the analysis of the curriculum. For this purpose, we use an example a current project on the analysis of competencies in Spanish university degrees. As a result of this study, we indicate and evaluate the steps to collect the data and we offer some possibilities of analysis. We insist on the high probability of making systematic errors, we suggest how to reduce it, and we offer proposals to reduce the work time invested as well. In the procedure, we confirm the convenience of training the research team by working in pairs, as it contributes to make the time invested in collection and analysis more profitable. We understand that curricular research serves to improve the structure of teaching practices, which is why we share this experience since it is necessary to apply an adequate and rigorous methodology to achieve the research objectives.
\end{abstract}

Keywords:

Analysis of curriculum, methodology, higher education, competencies

\section{Resumen:}

El objetivo de este artículo es ofrecer alternativas a una serie de inquietudes metodológicas para el análisis del currículum. Con este propósito, utilizamos como ejemplo un estudio en proceso sobre el análisis de competencias en titulaciones de universidades españolas. A raíz de este estudio, se indican y valoran los pasos para recopilar los datos y se ofrecen algunas posibilidades de análisis. Se insiste en que existe una alta probabilidad de cometer errores sistemáticos, indicamos cómo reducirlos y sugerimos propuestas para disminuir también el tiempo de trabajo invertido. En el procedimiento se comprobó la conveniencia de entrenar al equipo de investigación en la colaboración por parejas, ya que contribuye a rentabilizar el tiempo invertido para acopio y análisis. Entendemos que el análisis curricular sirve para mejorar la estructura de las prácticas docentes, por eso compartimos esta experiencia, ya que es necesario aplicar una metodología adecuada y rigurosa para lograr los objetivos de investigación.

\section{Palabras Clave:}

Análisis del currículum, metodología, educación superior, competencias

\section{Introducción}

Los estudios sobre el currículum aportan un conocimiento acerca de la planificación educativa. Es importante analizar los planes de estudios para mejorar la estructura de las prácticas docentes, pero también conviene estudiarlos con cierto rigor metodológico. De lo contrario, las conclusiones a las que lleguemos no serán confiables y, por tanto, no serán tan útiles para planificar la docencia en una determinada materia o asignatura.

Este artículo trata sobre la metodología para el análisis del currículum. El objetivo es ofrecer opciones metodológicas para el acopio de datos y su análisis, a fin de evitar problemas de rigor y errores en el procedimiento. Por tanto, este trabajo no ofrece resultados de investigación, sino procedimientos para el acopio y organización de los

\footnotetext{
a Universidad Autónoma del Estado de Morelos, https://orcid.org/0000-0003-2005-3436, Email: anaescalante7@ hotmail.com

b Autor de Correspondencia, Universidad de Valencia, España y Universidad Autónoma del Estado de Morelos, México, https://orcid.org/0000-0001-5342-7137 Email: emocra@alumni.uv.es

c Universidad de Valencia, España, https://orcid.org/0000-0002-6267-0080, Email: garfran9@uv.es
} 
datos. Se incluyen algunas opciones para llevar a cabo el análisis, pero no se aplica el procedimiento de análisis de los datos, ya que esto requeriría un artículo diferente para cada tipo de análisis. Con esta idea, esbozamos el procedimiento de una investigación en proceso.

El estudio sirve como un ejemplo para guiar al lector en el acopio de los datos y propuestas para su análisis; está situado en el ámbito de la educación superior y trata sobre el análisis de competencias. Se incluyen las titu laciones de Pedagogía e Ingeniería en Telecomunicaciones que se ofertan en las universidades españolas, tanto públicas como privadas. Seleccionamos estas áreas de estudio, ya que nos interesa la formación de pedagogos y para contrastar los resultados, se seleccionó de manera aleatoria la titulación de Ingeniería en Telecomunicaciones. La Ingeniería en telecomunicaciones tiene una estructura diferente a la titulación de pedagogía y pertenece a un área de conocimiento distinta.

Con este estudio como ejemplo, valoramos el proceso de acopio de datos y ofrecemos posibilidades de análisis, que pueden ser extensibles a otros estudios sobre cu rrículum. Mostramos cómo acceder a los datos y cómo plantear una estrategia para recopilarlos y registrarlos correctamente. Indagamos en el efecto de repartir las tareas y entrenar al equipo de investigación para desempeñar procedimientos específicos, obteniendo variables directas e indirectas.

En la estrategia para el acopio de datos indicamos procesos que evitan errores sistemáticos y ayu dan a ahorrar tiempo. A lo largo del artículo debatimos las limitaciones de este tipo de estudios sobre el currículum, entre otras ideas de interés.

\section{Análisis del currículum}

La teoría del currículum incluye varios ejes de análisis que se han mantenido desde las teorías tradicionales del siglo pasado (Gimeno Sacristán, 1981). Algunos de estos ejes son: los objetivos, los contenidos y los métodos de enseñanza y, la evaluación para saber si se han cu mplido los objetivos que se previeron en una determinada materia. Las últimas tendencias acerca del currículum universitario ponen de relieve la disrupción como forma de planificación docente (Samuel Michael, Dhunpath \& Amin, 2016; Quinn, 2019), pero los planes de estudios disponibles para analizar mantienen en gran medida el esquema tradicional, de modo que nos basamos en las teorías clásicas para el análisis del currículum.

En este orden de ideas, mostramos cómo podría realizarse el acopio de los datos y las opciones de análisis del currículum que surgen a partir de estos datos. El procedimiento que compartimos se origina en un estudio del currículum en las universidades españolas, por lo que conviene clarificar algunas particularidades de este contexto con el fin de evitar confusiones.

El sistema universitario español tiene ciertas particu laridades, aunque está adscrito al Proceso de Bolonia, en el Espacio Eu ropeo de Educación Superior. Los profesores planifican las asignaturas de los planes de estudios en los programas docentes o "guías docentes", que se redactan sobre la base de un modelo preestablecido. Este modelo trae incorporados por lo general los siguientes apartados:

- Resumen de la asignatura.

- Conocimientos previos que se requieren.

- Competencias por desarrollar.

- Resultados de aprendizaje.

- Descripción de los contenidos que se enseñan.

- Descripción de las actividades formativas.

- Volumen del trabajo en tiempos.

- Metodología de enseñanza.

- Evaluación de los aprendizajes.

- Referencias para estudiar la asignatura.

Como decíamos, este esquema parece ajustarse más a la visión clásica del currículum que a la flexibilidad que están proponiendo algunos autores. Esto no implica renunciar a la disrupción de los programas para darles una forma más dinámica y espontánea, manteniendo cierto control en los objetivos de las asignaturas, los contenidos que se deben aprender y los criterios para evaluar el aprendizaje. Con la disrupción incluida, el modelo de las guías docentes está ajustado a los objetivos (las competencias y resultados de aprendizaje), a los contenidos, al método de enseñanza y a la evaluación. Por tanto, el análisis que proponemos está basado en estos ejes curriculares.

Seleccionamos uno de estos ejes para analizarlo con más detalle. Para el ejemplo que utilizamos sólo recabamos los datos relativos a las competencias, que formarían parte de los objetivos de las asignaturas. Los diseños curriculares basados en competencias están creciendo en interés y en importancia, al menos durante la última década (Gilmer Echols, Neely \& Dusick, 2018; Gargallo López, 2017). Es más, los programas docentes basados en tiempos se quedaron obsoletos para verificar los resultados de aprendizaje y los programas don de los estudiantes muestran habilidades y conocimientos parecen ser más adecuados (Kelly \& Colombus, 2016).

No obstante, antes de plantear la enseñanza, aprendizaje y evaluación de un contenido, es necesario definir ese contenido. Aquí reside parte de la importancia que se le atribuye a las competencias. La formulación de las competencias condiciona profundamente los planteamientos docentes, ya que viene acompañada de una in clinación hacia uno u otro objetivo, siempre en el sentido de que los estudiantes demuestren lo que aprendieron. Es por 
esa razón que el análisis de las competencias que vienen en las guías docentes despertó nuestro interés.

Hay fundamentalmente dos tipos de competencias, si las consideramos para su aplicación en un área determinada de estudio. Las competencias que se aplican solamente a un área de estudio suelen adjetivarse como "específicas" o "disciplinares", y las que se aplican a cualquier área son "genéricas" o "transversales". Nosotros utilizaremos en el artículo los términos "disciplinar" y "transversal" para evitar confundir al lector, puesto que el ejemplo que mostramos consiste en el análisis del tipo de competencia en función de su aplicación.

La diferencia entre formular una competencia disciplinar y formular una transversal es que la primera se ajusta a un perfil de empleo y la segunda va más allá, ya que es para la vida. Si tomamos la competencia "evaluar instituciones educativas", podemos aplicarla en las instituciones educativas, pero no fuera de ellas. En cambio, si tomamos la competencia "razonar con criterio" o "razonamiento crítico", podríamos aplicarla además en la vida diaria. Por lo general, las competencias de tipo disciplinar son más técnicas, más instrumentales y se aplican más a la práctica profesional, mientras que las de tipo tran sversal son orientativas.

En el caso que hemos visto, sería posible razonar con criterio sobre la evaluación en las instituciones educativas, pero no sería tan verosímil evaluar cómo fu ncionan las instituciones para alcanzar un razonamiento crítico en sentido amplio. Es más, evaluar el funcionamiento de una institución significa clasificarlo con criterios de evaluación en diferentes categorías, cuyo reduccionismo más abstracto sería: funciona "bien" o "mal". La decisión acerca de si una institución funciona bien o no se basa en un criterio para decidir cómo funciona. Tal vez no funciona bien porque se ha decidido que debe producir cierto número de egresados con un expediente académico positivo y si no lo hace, entonces funciona mal. De cualquier forma, evaluar conlleva el razonamiento crítico, pero razonar con criterio no implica necesariamente evaluar las instituciones educativas. Por lo tanto, no todo aquél que puede razonar con criterio es apto para dedicarse a la dirección o a la inspección de instituciones educativas, pero aquellos que van a ser directores o inspectores sí necesitan saber cómo razonar con criterio. Por eso nos interesa mostrar opciones metodológicas, por ejemplo, para saber qué tanto se prevén competencias de uno u otro tipo, y si existe un equilibrio entre lo disciplinar y lo transversal.

Con todo, entendemos que las competencias disciplinares podrían incluir contenido transversal, pero no así al revés, ya que las transversales se caracterizan por una ausencia de contenido disciplinar. La Figura 1 esboza esta idea, de acuerdo con la cual sería correcto formular competencias como "evaluar instituciones educativas con razonamiento crítico", pero no sería tan correcto formular competencias como "evaluar instituciones educativas para razonar sobre cualquier otra cosa de manera crítica”.

Regresando a la estructura de las guías docentes que se redactan en las universidades españolas, creemos que es necesario profundizar algo más en el contexto de estudio. No todos los sistemas universitarios funcionan igual y los estudios que se desarrollan en un sistema podrían no ser factibles en otro. Antes de diseñar un estudio semejante a lo que hicimos en nuestro ejemplo, instamos a los lectores a considerar el sistema educativo que van a analizar. Una particularidad del sistema universitario español es que las guías docentes su elen publicarse y están disponibles para estudio, pero esto no ocurre en todos los sistemas y en el sistema universitario de otro país quizás no hay acceso a los programas docentes. Tomar en consideración estas y otras características del contexto ahorra tiempo y esfuerzo en el análisis curricular.

\section{Figura 1. Formulación de las competencias.}

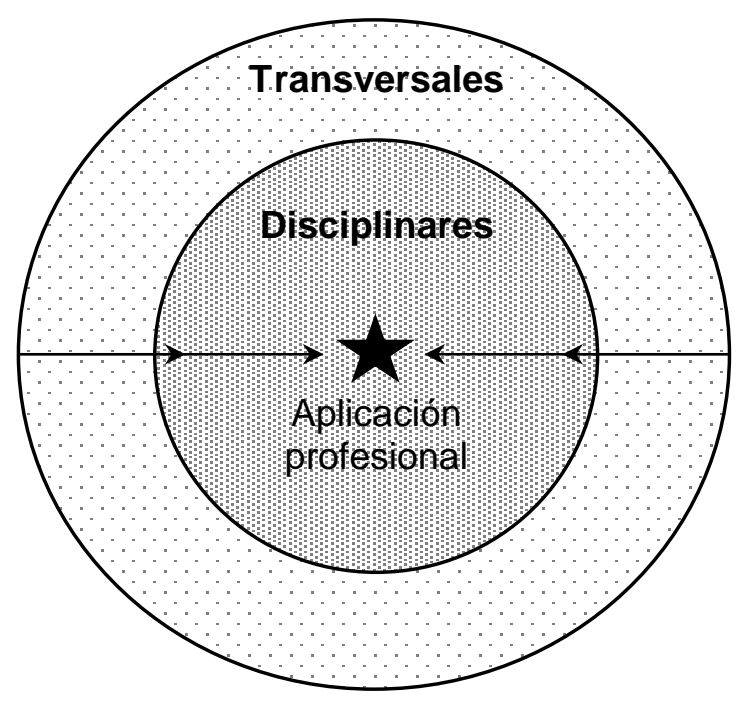

Fuente: Elaboración propia.

\section{Posibilidad de realizar el estudio}

En el contexto de nuestro estudio, la Agencia Nacional de Evaluación de la Calidad y Acreditación (ANECA) verifica las guías docentes para garantizar la formación del perfil de egreso de cada titulación. Las universidades publican las guías docentes con los detalles de una asignatura, así como toda la información fundamental necesaria para su seguimiento. Las publican porque las guías constituyen el contrato de aprendizaje entre la oferta de una universidad y los estudiantes que se matriculan en dicha universidad (San Martín Gutiérrez, Jiménez Torres \& Jerónimo Sánchez-Beato, 2016). Los estudiantes de- 
ben tener la posibilidad de conocer los detalles del contrato antes de pagar su matrícula.

En cada universidad se siguen ciertos procedimientos. En su mayoría, consisten en una discusión colegiada acerca de la formulación de las competencias. Las competencias se concretan con el logro de un consenso, que es avalado por las instancias de calidad de cada universidad.

Nosotros tomamos los datos sobre las competencias que ofrecen las guías docentes de los títulos de Pedagogía y de Telecomunicaciones en todas las universidades españolas. La intención de contrastar las competencias según su aplicación (transversal o disciplinar) fue determinar si estas competencias permiten a los egresados de las dos titulaciones desenvolverse de forma congruente con la disciplina. Es decir, si permiten atender los problemas de cada disciplina, dando también una formación integral y de alta calidad científica, tecnológica y humanista, propia de cualquier área.

Finalmente, la muestra del estudio estuvo compuesta por 37 programas de titulación: 23 de Pedagogía y 14 de Telecomunicaciones. Logramos acceder a las guías docentes de 1,896 asignaturas: 1,032 de Pedagogía (54.4\%) y 864 de Telecomunicaciones (45.6\%). Las guías que incluimos para el estudio suponían el $98.33 \%$ de las guías docentes de todos los programas de ambas titulaciones, así que no fue necesario calcular el tamaño muestral requerido para saber que los resultados serían representativos, al menos para las dos titulaciones que se analizaron.

En la titulación de Pedagogía, recabamos información de 5,520 créditos European Credit Transfer System (ECTS), lo que corresponde a 138,000 horas de formación para el alumnado. En Telecomunicaciones fueron 3,600 créditos, que implican 90,000 horas de formación. En total obtu vimos datos sobre las competencias previstas en 228,000 horas. Con todas las guías docentes, analizamos un total de 20,321 competencias. Los detalles están disponibles a continuación en la Tabla 1.

Insistimos en la relevancia de considerar la posibilidad de llevar a cabo el estudio, debido a la disponibilidad de la información. Si no hay datos, no tenemos estudio. El acceso a los datos es fundamental, pero es distinto en cada sistema educativo. Cuando se trata de análisis curricular, es muy importante contar con la información del eje cu rricular que vamos a analizar.
Tabla 1. Competencias recabadas para el estudio

\begin{tabular}{llll}
\hline Titulación & Disciplinar & Transversal & Total \\
\hline Pedagogía & 7,888 & 4,865 & 12,753 \\
Telecomunicaciones & 4,538 & 3,030 & 7,568 \\
Total & 12,426 & 7,895 & 20,321 \\
\hline
\end{tabular}

Fuente: Elaboración propia con los datos recabados.

\section{Acceso a los datos}

Investigar implica un proceso riguroso y ordenado, basado fundamentalmente en una población delimitada, el acopio de información sobre una muestra de dicha población, el análisis de los datos y el establecimiento de conclusiones. La población de un estudio constituye el conjunto total de unidades de análisis, sean personas u objetos, con unas características comunes que podemos observar de forma directa o indirecta. La observación de estas características o datos debe ser rigurosa y orden ada para que todo el proceso lo sea también.

Existen bases de datos con información ordenada que dan acceso directo a los datos necesarios para desarrollar un determinado estudio. Las bases suelen estar indexadas y cumplen criterios de calidad, de modo que los datos llegan a ser confiables. Usamos bases con estas características para obtener un conjunto de datos ordenados sobre una unidad de estudio. En nuestro ejemplo, esa unidad que se somete a estudio son los títulos de Pedagogía y Telecomunicaciones, y más concretamente, las competencias de las guías docentes de sus asignaturas.

Cuando analizamos el currículum, los datos de interés son diferentes a los que se analizan en otros estudios. Aquí se analiza la información relativa a los ejes de los que hemos hablado al inicio del artículo, o a otros ejes parecidos, pero en cualquier caso se analizan aspectos de la planificación o programación de la docen cia en un nivel y en un sistema educativos. El currículum es una planificación, un plan, un proyecto docente que se llevará a cabo en un tiempo que se ha delimitado para la ocasión de aplicar un proceso de enseñanza-aprendizaje concreto. Por tanto, no se trata de analizar el éxito de un método de enseñanza, es más bien el análisis sobre cómo se prevé aplicar dicho método y sus características: si está basado en evidencias empíricas, si es adecuado para enseñar lo que se pretende enseñar, si puede enmarcarse en una orientación docente, como una enseñanza centrada en los estudiantes, etcétera. Es más, incluso cuando se termina la formación universitaria y los estudiantes egresan, el currículum sigue siendo poco más que una aproximación a lo que se supone 
sabe hacer con un título universitario. Sabemos que el título que cursaron los egresados tenía previstas ciertas horas de formación y las tenía previstas de manera ordenada, distribuyéndolas por asignaturas. Sin embargo, nadie asegura que el egresado haya trabajado exactamente ese número de horas, sobre todo cuando parte de este tiempo se reserva para estudiar en un ámbito informal de aprendizaje, como el hogar; por lo menos en el contexto de las universidades españolas.

Los datos que nos interesan para el análisis curricular son sobre la programación docente y, de hecho, muchas veces existen bases de datos con información específica acerca de los programas o proyectos docentes. Esto suele depender del sistema educativo y del nivel de ese sistema. En el caso de nuestro ejemplo, sí hay una base de datos específica que reúne información especialmente relevante para nuestro estudio. En otros contextos de estudio, sería conveniente explorar si existen este tipo de bases y hacer uso de ellas si están disponibles. También es interesante consultar las bases de datos convencionales, que reúnen por lo general los hallazgos de investigaciones concluidas. De hecho, es necesario consultar estas bases para armar un estado del arte acerca del estudio que se va a realizar, pero si no se trata de un meta-análisis o un estudio similar, los datos que se analizarán deberían extraerse de bases como la utilizada en este trabajo, y que presentamos más adelante en este mismo apartado.

Particularmente, cuando no se tiene experiencia en el análisis del currículum, es conveniente buscar artículos publicados en torno a nuestro contexto de estudio, con el fin de observar sus apartados de metodología y ver cómo procedieron. Entre otros detalles, esto permite conocer las bases de datos específicas, cómo acceder a ellas y cómo utilizarlas para obtener información sobre la población que queremos analizar. En el ejemplo que estamos mostrando nos basamos en el procedimiento de estudios previos (García-García, López-Torrijo \& SantanaHernández, 2020).

Nosotros accedimos a un repositorio público desarrollado por el Ministerio de Ciencia, Innovación y Universidades de España, llamado Registro de Universidades, Centros y Títulos (RUCT). El repositorio contiene información detallada en línea acerca del sistema universitario español. Al ingresar a su página web oficial, nos dirigimos a la sección de universidades y posteriormente, a la sección de títulos. Llenamos los campos de acceso, incluyendo "Pedagogía" en la denominación del título, "grado" en el nivel académico y en la rama de conocimiento, "ciencias sociales y jurídicas". En el caso de Telecomun icaciones agregamos "Telecomunicación" en la denominación del título, "grado" en el nivel académico, e "Ingeniería y Arquitectura" en la rama del conocimiento. La plataforma arrojó un listado con todas las titulaciones de Pedagogía y Telecomunicaciones ofertadas en España y la universidad donde se ofrecían.

Consideramos que el RUCT es una base de datos donde pudimos recabar información confiable sobre la unidad de estudio que íbamos a investigar, ya que se trata de datos oficiales y actualizados.

Después de realizar la búsqueda, el listado de titulaciones ofertadas para estudiar Telecomunicaciones tenía ciertas características. Algunas titulaciones estaban orientadas a los sistemas de telecomunicación, otras a la telemática, en algunos casos eran especialidades de informática, y no se podía establecer una homogeneidad del título. Por lo tanto, decidimos tomar solamente los títulos de Telecomunicaciones orientados a estudiar los sistemas de telecomunicación. En el caso de Pedagogía, todos los títulos eran muy parecidos o eran iguales.

Una vez detectadas las universidades, sistematizamos la información en una hoja en Microsoft Excel, para identificar el número de centros que ofertan estas titulaciones. Posteriormente, con este listado accedimos a las páginas web oficiales de cada universidad y recuperamos todas las guías docentes disponibles para cada asign atu ra de ambas titulaciones. Las descargamos y las ordenamos en carpetas por curso anual (de $1^{\circ}$ a $4^{\circ}$ curso), por semestre, por titulación y por universidad, de manera in tercalada y coherente, como si estuviéramos editando una tabla con categorías de datos (ver Tabla 1 para un ejemplo).

\section{Estrategia para el acopio y organización de datos}

Una vez que se accede a los datos, es conveniente filtrarlos y capturarlos con detalle. Es importante establecer criterios para la inclusión y exclusión de los datos a los que se pudo acceder, vaciarlos correctamente en softwares adecuados y de manera estratégica, considerando el análisis, limpiar la base de datos que resultó del procedimiento del acopio y transferir la información a otros programas de análisis, si fuera necesario. A continuación, mostramos cuál fue la estrategia para el acopio de datos que seguimos en el estudio sobre las competencias.

\section{Criterios de inclusión y exclusión}

El acopio de datos requiere tiempo y esfuerzo, y conviene sistematizarlo para no repetir más adelante el proceso. Un modo de sistematizarlo es decidir qué datos nos interesan para el estudio que vamos a realizar y qué datos no. Dicho de otra forma, conviene definir con claridad los datos que estamos incluyendo y los que estamos excluyendo para el análisis. Si establecemos criterios de inclusión y exclusión de los datos, otros podrán replicar el estudio y será posible contrastar los hallazgos. De he- 
cho, la replicabilidad es uno de los beneficios de utilizar criterios para seleccionar datos antes del análisis y además, sirve para delimitar el universo de medida o población a la que pertenece una muestra.

Habitualmente, los criterios de inclusión y exclusión hacen referencia a los participantes de un estudio. En el ejemplo que estamos mostrando los aplicamos a las competencias de las guías docentes, ya que son las unidades que están siendo sometidas a estudio, aunque no sean personas. En cualquier caso, podemos considerar los criterios como un conjunto de características predefinidas que sirven para la identificación de sujetos, documentos, o cualquier unidad que forme parte de una muestra, y que será incluida en un estudio de investigación (Salkind, 2010). Nosotros utilizamos los criterios para excluir algunas competencias del análisis y para clasificar las que finalmente íbamos a analizar, ya que habían sido incluidas.

Excluimos las competencias con una definición imprecisa, que podía provocar múltiples interpretaciones (criterio 1), como "creatividad" o "comunicación", dado que no dilucidan qué significan. En el caso de la comu nicación, por ejemplo, no se precisa si la competencia incorpora habilidades para redactar textos con cierto rigor técnico y científico, o si se trata de exponer trabajos académicos oralmente ante diferentes auditorios. Esta competencia se presta a confusión y no podríamos asegurar qué estamos analizando, por lo tanto, decidimos excluirla. También excluimos las competencias que consistían en la realización de actividades (criterio 2), como un "ejercicio original a realizar individualmente y presentar ante un tribunal". Ese ejercicio es una actividad, pero no es un resultado de aprendizaje, aunque haya sido pensado para aprender algo. Por eso no lo consideramos como parte del análisis de datos.

Una vez excluidas ciertas competencias, clasificamos las que habían sido incluidas en competencias disciplinares y transversales. Consideramos que las disciplinares, si bien podrían requerir contenido transversal-como las TIC-, se aplican directamente a cuestiones propias de la disciplina (criterio 3), de modo que son específicas de una profesión. En cambio, las transversales sólo requieren un contenido genérico, transversal (criterio 4). Esto nos dio un concepto personalizado sobre lo que entendemos por competencia transversal y por competencia disciplinar.

Supongamos que otro equipo de investigación analiza las competencias de los planes de estudios, que es el mismo universo de medida o la misma población que analizamos en nuestro ejemplo. Si este otro equipo tiene razones para creer que nuestros resultados son equívocos, necesitará replicar el mismo estudio y no podrá hacerlo si no conoce qué competencias excluimos del análisis y cómo clasificamos las que sí incluimos. Si na- die puede replicar nuestro estudio, será difícil corroborar la validez y confiabilidad de los resultados que obtuvimos (The National Academies of Sciences, Engineering, and Medicine, 2019). Una forma de evitarlo es utilizar criterios de inclusión y exclusión.

\section{Registro de los datos}

La codificación en el software Atlas.ti fue otro filtro para los datos. Durante los pasados meses de abril a julio de 2020 se realizó la concentración de 1,896 guías docentes, correspondientes a los títulos de Pedagogía e Ingeniería en Telecomunicaciones ofrecidos por 32 u n iversidades de España. Al principio contemplamos 37 instituciones, que sumaban 2002 guías, sin embargo, cuatro de ellas no tenían disponibles los documentos para con sultarlos en línea. Además, algunas guías docentes no tenían los elementos suficientes para codificarlas, ya que no se especificaban las competencias.

Para llevar a cabo la codificación, se creó un listado de las competencias que aparecían en cada una de las guías. Con base en este, fuimos encontrando las similitudes y diferencias que permitieran codificar las competencias con el mismo procedimiento en todos los documentos que componían la unidad hermenéutica.

Se partió de una lista que incluía 33 códigos elaborados a priori, contando con los datos de identificación: Titulación (título al que correspondía una guía), Créditos (número de créditos ECTS de una asignatura) y Asignatura (el nombre de la materia).

En cuanto a las competencias que se encontraban en las guías docentes, se indicó si eran transversales (CT), disciplinares (CD) o cuando no cumplieron con el criterio de inclusión se excluyeron.

En Microsoft Excel incorporamos un número de folio para cada guía docente. El número de folio permite ordenar los datos en función de una determinada variable e identificar el orden original en el que se registraron esos datos. Saber el orden original permite, en cualquier momento, identificar casos (guías docentes particulares), si manejamos más de un archivo. A veces nos interesa trabajar con los datos en función de una determinada variable y los ordenamos para identificar mejor esa variable. Supongamos que la variable que estamos contrastando es la titulación y queremos ver claramente si las guías son de Telecomunicaciones o son de Pedagogía. Entonces podemos ordenar el conjunto de datos con todas las guías de una titulación primero y con las de la otra titulación después. Para regresar al orden de los datos que teníamos al inicio, disponemos del número de folio y podemos repetir este procedimiento tanto como sea necesario durante la fase de análisis.

Además, utilizar número de folio reduce la probabilidad de cometer errores sistemáticos durante el proceso de acopio de los datos. Los errores sistemáticos son aque- 
llos que se deben al diseño o al análisis de los datos, y comprometen la validez de los resultados (Rabinovich, 2005). Si los datos que recopilamos se pueden interpretar y analizar de manera errónea, habrá problemas para confiar en que estamos analizando lo que pretendemos analizar realmente.

Usando número de folio nos aseguramos en mayor medida de que los casos que estamos analizando son los que pretendíamos analizar en una determinada fase del estudio. Es decir, si estamos analizando los diez primeros casos, correremos un riesgo menor de que el caso que suponemos como el número 1, 2, 3, etc., no sea en realidad el caso número $15,16,17$, etc., sin que nos hayamos dado cuenta por error. El factor humano es la causa de los errores de tipo sistemático, como elaborar un diseño de investigación que no corresponde con lo que se pretende investigar o recopilar los datos de una manera poco clara, que se preste a confusión durante el análisis.

Con la misma idea, al trasladar los datos en Microsoft Excel utilizamos celdas auto-calculables en lugar de calcular las variables en un papel e incorporar luego el resultado en su celda correspondiente. Esto contribuye a cometer menos errores sistemáticos igualmente. Más adelante hablamos sobre cálculo de variables indirectas y la conveniencia de utilizar celdas auto-calculables para obtener los puntajes.

\section{Entrenamiento para el trabajo en parejas}

Trabajar en parejas contribuye a mejorar los tiempos en el acopio de datos, además de aminorar el riesgo de cometer errores sistemáticos. Sin embargo, conviene invertir en un entrenamiento específico para las parejas de trabajo, que supone invertir un tiempo al inicio del vaciado de los datos. Este tiempo de entrenamiento contribuirá más adelante a reducir el tiempo de esta fase del proceso de acopio y para demostrarlo, nosotros comenzamos a capturar los datos sin entrenar a la pareja de trabajo. Cuando llevábamos el $20 \%$ de los datos vaciados en Microsoft Excel y en Atlas ti, la pareja que estaba cargando los datos en los softwares recibió un entrenamiento. Vaciamos los datos en estas dos fases (una sin entrenamiento previo y otra con él) porque intuimos que el entrenamiento mejoraría los tiempos, pero no lo habíamos leído ni estudiado antes en otros archivos sobre metodología de investigación. Por tanto, decidimos probar nosotros mismos nuestra intuición y dio resultados positivos, que efectivamente reducen el tiempo del acopio en esta fase del vaciado de los datos.

Mientras no hubo un entrenamiento, la pareja no tenía un reparto de funciones explícito, y cada investigador se veía obligado a revisar los criterios para el vaciado de los datos en los softwares. Los criterios se revisaban con frecuencia cuando uno de los miembros del equipo hacía varios días que no trabajaba en ciertas categorías, como por ejemplo, la transversalidad de una competencia, o la optatividad de las asignaturas. También ocurría a menu do cuando no se comprendía bien un criterio para clasificar competencias, ya que esto sembraba la duda en el investigador sobre si la clasificación que estaba realizando era o no correcta, y si debía reconsiderarla y volver a clasificar la competencia.

Cuando la pareja de trabajo fue entrenada, cada persona se especializó en unas tareas y dejó otras para su pareja. En nuestro ejemplo hubo dos parejas de trabajo: una que se encargó de la codificación en Atlas ti y otra que capturó los datos en Microsoft Excel. Mostramos a continuación el proceso de la pareja que capturó los datos en Excel.

Uno de los investigadores de esta pareja era responsable de trasladar las codificaciones que había realizado previamente la otra pareja en Atlas ti. Para el traslado había que contabilizar las competencias de tipo disciplinary transversal, registrarlas, asegurarse de que el conteo era correcto y cotejarlo con su pareja de trabajo. EI otro investigador de la pareja debía capturar estos datos en Microsoft Excel, asegurándose de que correspondían con los datos de la misma asignatura en una sola fila de la hoja de cálculo. El tiempo que tardó esta pareja en trasladar los datos a Excel fue significativamente men or después de entrenarse que al inicio del proceso $(Z=-$ $4.624, p=.000$ ), como se desprende de la Prueba de los rangos con signo de Wilcoxon (Wilcoxon, 1945), obteniendo la puntuación $Z$ con base en los rangos positivos. Se puede consultar un polígono de frecuencias en la Figura 2 sobre el proceso antes y después del entrenamiento por parejas.

El experimento que realizamos para contrastar la hipótesis según la cual el tiempo de procesamiento de los datos es significativamente menor después de un en trenamiento se llevó a cabo con una muestra de competencias. La unidad de análisis con la que trabajamos en el estudio de nuestro ejemplo son las competencias y pretendemos determinar si se trata de competencias disciplinares o transversales, de forma que el análisis de datos trate sobre la aplicación de las competencias que vienen en las guías docentes. En este sentido, tomamos una muestra de 28 guías antes del entrenamiento y de otras 28 después de entrenar al equipo de trabajo. En las guías que tomamos antes de que la pareja se entrenase había 278 competencias a procesar y en las guías que tomamos después, había 509. Cuando medimos el tiempo, consideramos cuánto tardó la pareja en procesar cada competencia. 
Figura 2. Efecto del entrenamiento porparejas

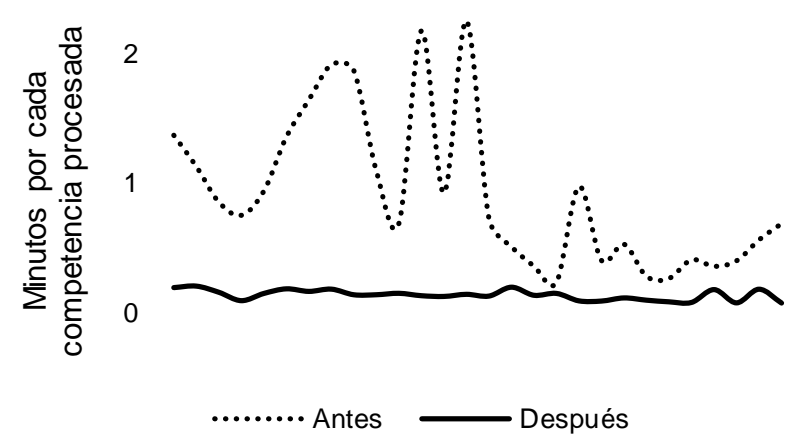

Fuente: Elaboración propia.

Antes del entrenamiento, el promedio en minutos por cada competencia fue 0.92 , con desviación típica igual a 0.6 . En el momento posterior al entrenamiento la media fue 0.13 , con una desviación de 0.04 , lo que evidencia una mejora del tiempo de procesamiento. Por ese motivo, recomendamos el entrenamiento para el trabajo en parejas.

\section{Tiempo de categorización de los datos}

Con todo, nosotros tardamos 81 días en procesar todo el paquete de datos, excepto el primer $11.42 \%$ de los datos que, utilizamos para situarnos en el procesamiento y tomar decisiones para repartir el trabajo (Figura 3). Este tiempo no sólo incluye el ejemplo que estamos utilizando sobre la categorización de estas competencias como disciplinares y transversales. Entraña además procesamientos que no estamos incluyendo en el ejemplo. Sin embargo, creemos que mostrar el tiempo que tardamos en procesar el set de datos con dos parejas de trabajo ofrece una idea sobre el tiempo que se puede tardar en procesar los datos para un análisis similar. Esto podría servir de ayuda para planificar otros estudios o proyectos de investigación. Tal vez también sea útil para los estudiantes que están planteando sus tesis de licenciatura, maestría o doctorado.

En la gráfica se observan momentos en los que hubo algo menos de actividad y momentos en los que se trabajó más duramente. Esto se debe a las agendas de cada persona que compone el equipo de investigación. Hay periodos en los que alguien tiene ciertas responsabilidades y no puede dedicar tanto tiempo al procesamiento de estos datos. En ocasiones, decidimos que es necesario un descanso porque identificamos cierto agotamiento y sabemos que es necesario detener dos o tres días la tarea de procesamiento. Otras veces sencillamente respetamos los periodos vacacionales o los días festivos. En cualquier caso, recomendamos estas pequeñas pausas porque son una ayuda a lo largo del proceso; no para ganar tiempos, como en el caso del entrenamiento por parejas, sino más bien para ganar en eficacia durante el procesamiento. Una mente agotada no percibe tantos errores al procesar datos como una mente despejada y descansada. Se trata de los errores sistemáticos $y$, ya que no se pueden evitar, se trata de reducirlos tanto como sea posible.

\section{Figura 3. Tiempo acumulado de procesamiento}

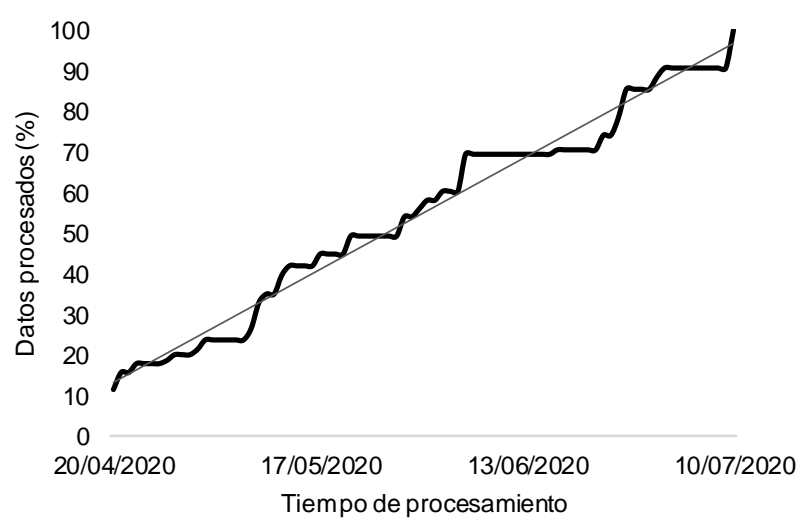

Fuente: Elaboración propia.

\section{Limpiado de la base de datos}

Después de registrar todos los datos, es relevante limpiar y depurar la base final. Esta fase de limpieza consiste en descubrir, corregir y/o eliminar los registros de datos que pudieron haber sido capturados con errores de entrada, incluso con errores ocasionados por fallos en el propio sistema de procedimiento. Es en esta fase cuando los investigadores deben identificar los datos incorrectos 0 incompletos para substituirlos, modificarlos o eliminarlos si es necesario. A estos datos erróneos los denominamos datos "sucios". Una base con datos sucios e incorrectos produce resultados viciados e imprecisos en el momento de realizar un análisis con cualquier software. Es por ello que es necesario revisar con detalle cada uno de los datos para mantener la calidad de éstos. La calidad de los datos puede valorarse con criterios como la exactitud, la consistencia, la uniformidad y la unicidad, entre otros. En nuestro estudio, la base de datos se limpió con dos miembros del equipo de investigación. Lo hicimos así porque una doble revisión ayuda a evitar la pérdida de información y cometer errores durante la corrección de los datos.

Antes de iniciar con la depuración, los dos miembros del equipo registraron las correcciones observadas para cada conjunto de datos en una lista. Revisar los datos por cada conjunto de estos contribuyó a tener un mayor control por secciones, sobre todo cuando se trata del manejo de una cantidad grande de datos. En nuestro 
estudio se revisaron los valores por universidad, y se verificó cada columna y fila para detectar cualquier dato anómalo. Posteriormente, se revisaron las celdas autocalculables para validar las fórmulas establecidas y se homologaron los valores a un solo formato. En el momento de finalizar con la limpieza de la base de datos es recomendable efectuar una copia de seguridad de todos los materiales. El resguardo de la información previene al equipo de trabajo ante sorpresas desagradables, como tener que iniciar el proceso otra vez porque se perdió la base de datos final y no se sabe cuál es la última versión disponible. Además de conseguir una copia de seguridad de la base final, conviene numerar las versiones de la base en cada una de sus modificaciones, así como la fecha de cada versión.

\section{Transferir la base de datos a otros softwares}

En muchas ocasiones, el software donde registramos los datos no ofrece las opciones de análisis que necesitamos para un estudio. Por eso, después de la revisión y limpieza de la base de datos final, a veces debemos transferir sus datos a un software de análisis más adecuado para el estudio que se va a realizar. El software debe seleccionarse en la fase de proyección del estudio, antes del acopio y el análisis de los datos. Existen varios softwares disponibles en el mercado, algunos de los cuales son de acceso libre. Conviene valorar un software que ofrezca los servicios que necesitamos para el análisis, incluso tener en mente otros materiales secundarios para prevenir las posibles limitaciones que no siempre se conocen al inicio, pero que muchas veces nos encontramos en la fase de análisis de manera imprevista.

Nosotros transportamos la base de datos desde Microsoft Excel a IBM SPSS Statistics. Para importar la información contenida en la hoja de cálculo Excel se requiere verificar con cautela que los datos en este archivo cumplan con los requerimientos estructurales del software al que vamos a transferir los datos, que en este caso es SPSS. Esto se hace para evitar inconvenientes, como perder más tiempo del necesario u obtener resultados erróneos y pensar que son certeros.

Hay tres parámetros que se deben considerar para iniciar el transvase de los datos: 1) su estructura, 2) el título de las columnas y 3 ) el rango. En la estructura de los ficheros de SPSS las variables vienen en las columnas y los casos o registros, en las filas. SPSS no incluye los títulos dentro de las celdas donde vienen los datos, por lo que el archivo a importar no debe contener títulos. Además, debemos tener en cuenta el rango de información útil que deseamos importar. Una vez importados los datos en SPSS, hay que definir las propiedades de cada variable. En nuestro caso, los valores que capturamos en Excel estaban registrados indicando los decimales con puntos, en tanto que la versión que utilizamos de SPSS trabaja con comas para indicar los decimales. Al analizar los datos, los resultados no fueron congruentes con lo que esperábamos. Este problema nos obligó a retroceder e identificar qué estaba ocurriendo con los valores iniciales, lo que nos hizo invertir más tiempo del esperado para el análisis. Para solucionar el problema, fue necesario regresar a la base de datos inicial, en Microsoft Excel, y convertir los datos para que los decimales estuvieran indicados con comas, no con puntos.

\section{Obtención de puntuaciones indirectas}

Antes de continuar con el análisis de los datos, durante el tiempo de acopio podemos registrar dos tipos de variables en función de su obtención: las que obten emos de forma directa, como la optatividad de las asignaturas o el número de créditos ECTS que ocupan en el global de la titulación a la que pertenecen; y las otras, que obtenemos indirectamente y necesitamos calcular sus puntajes, porque no podemos observarlos sino estimarlos.

No deben confundirse las variables o puntuaciones indirecta con las que se clasifican por medio de criterios de inclusión y exclusión, que se obtienen de manera directa. Por ejemplo, el criterio según el cual una competencia es disciplinar o es transversal nos ayuda a clasificar competencias, que son una variable directamente observable entre el conjunto de datos. Nosotros observamos una competencia en la guía docente y decidimos con un criterio preestablecido si esa competencia era disciplinar o transversal. El criterio actuó como si fuera una regla de medir con dos centímetros. Si la competencia alcanza el primer centímetro, pertenece a una categoría que denominamos transversal. Si alcanza el segundo cen tímetro, podría incluir contenido transversal en su definición, pero contiene de todos modos contenido disciplinar, y su aplicación llega específicamente hasta la actividad profesional de esa disciplina.

Podemos calcular variables que se obtuvieron de manera directa para obtener variables o puntuaciones indirectas. En nuestro estudio sobre competencias calculamos unas tasas que sirven para valorar el tamaño que ocupa cierto tipo de competencias, tomando como referencia el setde datos en su conjunto. Una de las tasas que calculamos fue la de transversalidad. La tasa fue igual al cociente del sumatorio de las competencias transversales entre el de competencias totales, ya sea para una asignatura, para un programa de titulación o para una determinada sección de la muestra.

Las puntuaciones indirectas suelen ser útiles para análisis cuantitativos. La tasa de transversalidad que obtuvimos en nuestro estudio constituye una variable continua, que permitirá calcular correlaciones parciales, realizar análisis de regresión, etc., junto con otras puntuaciones del mismo orden. Esto permite realizar análisis de va- 
rianza con base en un factor, como la titulación o la optatividad. En suma, ofrecen posibilidades de análisis más amplias que las que tenemos disponibles gen eralmente con las puntuaciones directas para el análisis curricular. Con el fin de reducir la probabilidad de cometer errores de tipo sistemático, recomendamos configurar celdas en una hoja de cálculo de Microsoft Excel, si se pretende emplear este programa. Otros softwares ofrecen servicios del mismo estilo, aunque con procedimiento diferente. En el paquete estadístico IBM SPSS Statistics encontramos las funciones de sintaxis o la opción de calcular una variable a partir de las variables que ya hay en el set de datos. De todas formas, recomendamos las celdas auto-calculables porque nos aseguramos de que todas las celdas reportan un valor numérico que siguió el mismo procedimiento para su cálculo que en el resto de celdas de la misma variable. Las celdas auto-calculables aportan validez al análisis por el procedimiento que se siguió en la fase de acopio de los datos.

\section{Posibilidades de análisis}

Los datos que recopilamos para nuestro estudio, que está sirviendo como ejemplo para el análisis curricular, ofrecen diferentes posibilidades de análisis, tanto cualitativo como cuantitativo.

\section{Análisis cualitativo}

Debido al propósito de las guías docentes, la redacción de estos documentos es concisa y generalmente incluye una acción que el estudiante debe ejecutar, el conocimiento a dominar, previo a la acción, y el contexto dónde se ejecuta dicha acción.

Una de las vías analíticas con enfoque cualitativo que podemos utilizar con esta clase de datos es la hermenéutica, ya que en este tipo de análisis la interpretación incluye no sólo lo gramatical, sino también el contexto histórico. La cautela que requiere este método man tiene al investigador alerta y consciente de la alteridad. Esto implica que, si una interpretación es correcta o in correcta, la decisión sobre la corrección reside finalmente en la perspectiva del otro, ya sea quien lee, quien proporciona información y/o quien escribe.

Recomendamos tres enfoques de la hermenéutica para el tipo de datos que se manejan en un análisis curricular como el que estamos llevando a cabo:

- Hermenéutica de validación u objetivista: asume que existen significados inmutables e inalterables, que son el objetivo de toda interpretación.

- Hermenéutica crítica: se valoran los significados de una expresión con base en ciertos criterios y a la luz de las condiciones históricas (Habermas, 2007).
- Hermenéutica filosófica: se halla el encuentro con un cierto contexto histórico o con las expresiones de otras personas (alter), y el interpretador juega un papel dialógico en el análisis (Gadamer, 1975).

En el primer tipo de análisis nos estaríamos ubicando en reconocer el acuerdo tácito del modelo por competencias y buscar en las guías docentes explícitamente lo que se espera del aprendiente al definir una competencia. En la hermenéutica crítica podemos encontrar la postura de la institución, o del profesorado y su compromiso éticopolítico con la función de la profesión en su en torno. En el marco del aprendizaje basado en competencias y del Proceso de Bolonia se trataría de formar a profesionales capaces de afrontar problemas o necesidades globales, y ya no estrictamente del área de influencia geográfica de las instituciones. Finalmente, desde una hermenéutica filosófica se pueden desentrañar aquellas cuestiones que hacen creíble lograr un perfil de egreso a partir de un conjunto de competencias, organizadas en las diferentes asignaturas de una titulación.

Otra posibilidad de análisis de textos, como las guías que analizamos en nuestro estudio, es el análisis de contenido cualitativo. Se trata de obtener de los textos su capacidad para contener aquello que permite llegar al conocimiento de distintos aspectos y fenómenos de la vida social. Una de las ventajas de este tipo de análisis es que percibimos a partir del texto, tanto el contenido manifiesto, -que es la representación y expresión del sentido que quien escribe pretende comunicar-como lo que hay latente, lo indirecto o aquello que utiliza al texto como un instrumento para expresar el sentido oculto del autor. Nuevamente, el contexto es lo que permite rescatar el sentido. Veamos un caso para comprender mejor el análisis de contenido.

En nuestro estudio, que sive como ejemplo, tomamos una competencia: "Analizar componentes y especificaciones para sistemas de comunicaciones guiadas y no guiadas por medios electromagnéticos, de radiofrecuencia u ópticos". Tomamos esta competencia de una guía docente de Ingeniería en Telecomunicaciones. Quizás para un lector las comunicaciones guiadas y no guiadas remitan a la idea de un mensaje que se transmite a alguien en particular o a un público amplio. Sin embargo, para el profesor que ha redactado esta competencia pensando en su asignatura y que tiene un dominio mayor en la materia que un presunto lector es innecesario especificar que se refiere a que estos medios de conducción sean alámbricos o inalámbricos.

Implícitamente, el autor del texto (el profesor) supone que los medios mencionados en la competencia requieren ciber-seguridad a su vez, y habrá que corroborar si el contenido de la asignatura hace referencia a cada tipo de medio al respecto. En cuanto al sentido pedagógico, el 
texto debe indicar la acción, que en este caso es analizar; el conocimiento para llevarla a cabo, que es conocer los componentes y especificaciones para sistemas de comunicaciones guiadas y no guiadas por los medios electromagnéticos, de radiofrecuencia u ópticos; y el contexto, que es el campo de las telecomunicaciones. Encontramos limitaciones en algunas metodologías cualitativas para analizar este tipo de textos, como es el caso de la fenomenología, la etnografía, o el análisis crítico del discurso, entre otros. Las competencias se representan en pequeños textos demasiados concretos como para realizar un análisis cualitativo en profundidad.

\section{Análisis cuantitativo}

Antes de proceder con el análisis cuantitativo, se deberían explorar los datos de la base que se introdujo en el fichero de SPSS, o en el software que se esté utilizando. Recordemos que explorar los datos nos ayuda a iden tificar errores sistemáticos, como un dato o una codificación erróneos. Detectamos también los valores atípicos o valores extremos, que se alejan de la media, la mediana, la moda, o cualquier tendencia central. Un valor extremo puede causar una dispersión demasiado grande en el conjunto de datos, de modo que podría ser interesante eliminar el caso con un valor atípico para reducir la dispersión y trabajar con una distribución menos asimétrica. Un análisis exploratorio nos permite localizar variables que en realidad son constantes y no existe variabilidad alguna, o bien variables con demasiados casos en una de las colas de la distribución -asimetría-, o sin valores alejados de las tendencias centrales -leptocurtosis-, entre otros posibles escenarios relacionados con la distribución de los datos.

Explorar los datos sirve también para generar baremos en función de un factor, como pueden ser las titulaciones en nuestro estudio: un baremo para Pedagogía y otro para la Ingeniería en Telecomunicaciones. Debería obtenerse la tendencia central y la dispersión para conocer cuál es la tendencia que representa mejor al conjunto de datos y el nivel al que puede representarlo. Por ejemplo, si tomamos un conjunto de datos con una distribución asimétrica, sería recomendable tomar como referencia la mediana, no tanto la media, y la dispersión que deberíamos calcular no es la desviación típica sino el rango intercuartílico, que es igual a $R Q=Q_{3}-Q_{1}$. Los percentiles se obtienen asimismo en un análisis exploratorio, previo a otros análisis posteriores, y son útiles para establecer los baremos y situar los casos que se analizan en un estudio estableciendo un grupo que podríamos denominar normativo. De esa forma se obtiene algo así como una regla de medir casos para valorar hasta qué nivel alcanza cada caso de un estudio.

Otra de las funcionalidades de los análisis exploratorios es el contraste de supuestos. Muchas pruebas estadísti- cas requieren el cumplimiento de ciertos supuestos, como los de normalidad, homogeneidad de varianzas o linealidad. Saber si cumplimos determinados supuestos o no permite tomar decisiones acerca del tipo de análisis que vamos a realizar. De hecho, cuando no se cu mplen los supuestos que requieren ciertas pruebas, conviene plantear un análisis no-paramétrico basado en rangos o en otras medidas más robustas.

Una vez que conocemos el set de datos, conviene diseñar una estrategia de análisis, puesto que el análisis es más complejo e implica un número mayor de posibilidades. Proponemos itinerarios posibles para el análisis cuantitativo, tras haber planteado orientaciones para un análisis hermenéutico de los textos en el subapartado de análisis cualitativo.

Dicho esto, uno de los itinerarios de análisis podría ser una comparación de promedios. Supongamos que tenemos la sospecha de que la titulación de Pedagogía e Ingeniería en Telecomunicaciones ofrecen tasas de transversalidad diferentes. Podríamos comparar los promedios en ambas titulaciones, calcular una prueba $t$ de Student, analizar la varianza (ANOVA) o, si no se cumplen los supuestos que requieren estas pruebas, contrastar la hipótesis según la cual ambas titulaciones son iguales en transversalidad de otra forma, como calculando pruebas no-paramétricas. En este caso sobre la tasa de transversalidad que presentan las dos titulaciones hay dos muestras independientes, una por cada titulación, de modo que se podría calcular alguna prueba noparamétrica como la $U$ de Mann-Whitney para contrastar la hipótesis.

Otro itinerario de análisis sería comprobar la consistencia interna. Sigamos con la tasa de transversalidad de nuestro estudio. Esta tasa es una variable continua, de modo que podría calcularse el Alfa de Cronbach para saber qué tan consistente es el puntaje en cada titulación y, por tanto, si es confiable o no. Sería deseable obtener una correlación alta entre los puntajes de los programas ofrecidos en las universidades españolas, porque eso daría un nivel alto de consistencia interna y permitiría confirmar la confiabilidad del constructo "transversalidad", conforme lo medimos en nuestro estudio. Cuando se analiza la consistencia intema de un puntaje, es importan te considerar el tipo de variable con la que estamos trabajando y si la prueba que vamos a aplicar requiere el cumplimiento de ciertos supuestos.

Otro diseño consistiría en tomar medidas repetidas con el fin de comprobar el efecto de un tratamiento en la muestra. En otras palabras, realizaríamos un test, aplicaríamos un tratamiento a la muestra y volveríamos a pasar el test, al que denominaríamos "re-test". Este diseño de estudio con la notación de Campbell y Stanley (1966) tendría este aspecto: $\mathrm{O}_{1}-\mathrm{X}_{1}-\mathrm{O}_{2}$. Pongamos un ejemplo concreto. Imaginemos que la exploración de los datos 
nos dio motivos para creer que las asignaturas optativas explican en cierto modo que las tasas de transversalidad sean mayores en Pedagogía que en la Ingeniería en Telecomunicaciones. Podríamos comprobar la influencia de las asignaturas optativas sobre la tasa de transversalidad si analizamos la diferencia entre las tasas de ambas titulaciones, sustraemos después las asignaturas optativas de ambas titulaciones -tratamiento- y volvemos a pasar la misma prueba para contrastar si hay igualdad o diferencia entre las tasas de transversalidad de los programas en ambas titulaciones. Si los resultados del test y del re-test no son equivalentes, las asign aturas optativas estarán influyendo en las tasas de transversalidad, incluso si el primer test no arroja los resultados que esperábamos, es decir, que las tasas de transversalidad fueran diferentes en una titulación y en la otra. Otro itinerario serían los estudios correlacionales. Podrían comprobarse las correlaciones entre variables categóricas con procedimientos como el Chi Cuadrado, o similares. En nuestro estudio tenemos diferentes variables categóricas. Tomemos dos variables dicotómicas: la aplicación de las competencias (disciplinar o transversal) y el título (Pedagogía o Ingeniería en Telecomunicaciones). Establecer la tabla de contingencia con estas dos variables daría paso a calcular la prueba Chi Cuadrada y determinar si existe o no una correlación entre el tipo de competencia y la titulación. También podrían comprobarse las correlaciones en tre variables continuas, no necesariamente siguiendo modelos lineales, aunque inicialmente sería recomendable hacerlo con un modelo lineal, sobre todo si el proyecto de estudio se encuentra en su fase exploratoria.

Los análisis correlacionales con variables continuas permiten realizar análisis de regresión para saber cuáles son las variables independientes que predicen la varianza de una variable dependiente. Por ejemplo, si calculásemos la tasa de optatividad de las titulaciones que analizamos en nuestro estudio, de manera similar a como calculamos la tasa de transversalidad, podríamos comprobar si existe un patrón de correlación entre ambas tasas y realizar análisis de regresión con modelos basados en el tipo de relación que exista entre las dos variables. Con este análisis sería posible conocer qué tanto la tasa de optatividad que tienen los programas de titulación predice la transversalidad en estos mismos programas.

Hay muchos más itinerarios de análisis, pero estos son los que podrían iniciar las primeras fases de análisis de datos, entre otros. Más adelante podrían realizarse análisis para saber cómo se agrupan las variables: análisis factoriales, o análisis discriminantes. Incluso sería interesante realizar análisis de conglomerados que, a diferencia de los análisis factorial y discriminante, no exigen cumplir el supuesto de linealidad, no requieren la distri- bución simétrica y además admiten variables categóricas. También sería conveniente realizar análisis lexicométricos o de minería de textos, ya que la muestra está compuesta por guías docentes, que son documentos institucionales con información curricular. Sin embargo, recomendamos utilizar otros softwares más sofisticados para un análisis de minería de textos, como el software R. En este artículo hemos mostrado ejemplos de nuestro estudio, utilizando Microsoft Excel y no Access, o IBM SPSS Statistics y no otros softwares más complejos. Lo hemos preferido así porque la pretensión, de este artícu lo, es aportar pautas metodológicas para iniciarse en el análisis del currículum, pero eso no excluye en absoluto la capacidad de esta línea de investigación para aventurarse hacia análisis más complejos, usando otros softwares con más beneficios.

\section{Conclusiones}

En este artículo plasmamos una serie de inquietudes metodológicas, que cualquier investigador novel enfrenta durante su carrera académica. La novedad y relevancia de este trabajo es la propuesta de utilizar la información procedente de datos disponibles en la red, que son valiosos para las investigaciones sobre el análisis cu rricular, pero que no parecen estar aprovechándose tanto como sería deseable.

El procedimiento descrito incluye pautas que permiten evitar problemas de rigor científico y ganar en validez y confiabilidad, a medida que se reducen los errores sistemáticos.

De acuerdo con la experiencia de los participantes en este trabajo, el entrenamiento por parejas aporta un ahorro del tiempo invertido para el acopio. Por este motivo, recomendamos un entrenamiento previo para el equipo de investigación sobre los aspectos que se sistematicen durante el acopio de datos. De esta forma, se consigue agilizar la captura de la información, particularmente en los estudios realizados en grupos de investigación o cuerpos académicos. Por otra parte, se evidenció la necesidad de realizar pausas para evitar el agotamiento y para mantener la eficiencia de los procesos de acopio y análisis, además, descansar reduce la probabilidad de cometer errores sistemáticos.

Concluimos que es estratégico utilizar criterios de inclusión y de exclusión, ya que no todos los datos son necesariamente útiles para una investigación.

Es necesario revisar la base de datos de cada investigación para garantizar que la información sea confiable, es decir, que los datos queden limpios y listos para analizar. Los datos que se utilizan tal como aparecen en la información recabada se denominan variables directas. EI investigador debe decidir si estos datos son suficientes para el estudio que pretende realizar o si necesita otros 
datos, obtenidos de manera indirecta por medio de un cálculo a partir de los datos disponibles.

En cuanto al objetivo de este artículo, aclaramos que no se ofrecen resultados de investigación, sino procedimientos para el acopio y organización de los datos. Por ello, ofrecemos algunas posibilidades para realizar análisis cualitativos y cuantitativos, aunque no desarrollamos aquí los detalles del procedimiento de análisis.

A partir de ciertas características de nuestro estudio, tratamos de orientar a los investigadores nóveles o en formación hacia la toma de decisiones para mejorar sus estrategias metodológicas.

La unidad de análisis del estudio, que aquí usamos como un ejemplo, surge de una trayectoria larga en cuanto a la teoría (Gimeno Sacristán, 1981; Samuel Michael, Dhunpath \& Amin, 2016; Quinn, 2019), pero tiene un recorrido breve en cuanto a la forma de recopilary analizar los datos. Hasta la fecha, no se han publicado muchos estudios sobre los programas o guías docentes de las universidades españolas (San Martín Gutiérrez, Jiménez Torres y Jerónimo Sánchez Beato, 2016; GarcíaGarcía, López-Torrijo \& Santana-Hernández, 2020). Por eso recomendamos promover el conocimiento y análisis de las guías docentes, teniendo en cuenta su importancia para los usuarios de la educación superior y la disponibilidad de los datos, que son de dominio público.

\section{Referencias}

Campbell, Donald T. y Stanley, Julian C. (1966). Experimental and quasiexperimental designs for research. Chicago, IL: Rand McNally.

Gadamer, Hans G. (1975). Verdad y Método I. Salamanca: Sígueme.

Garcia-Garcia, Fran J., López-Torrijo, Manuel y Santana-Hernández, Rafael. (2020). Educación inclusiva en la formación delprofesorado de Educación Secundaria: Los programas españoles. Revista de Currículum y Formación de Profesorado. Vol. 24, núm. 2, pp. 270-293. doi: $\underline{10.30827 / \text { profesorado.v24i2.14085 }}$

Gargallo, Bernardo (2017). Enseñanza centrada en el aprendizaje y diseño por competencias en la Universidad. Fundamentación, procedimientos y evidencias de aplicación e investigación. Valencia, España: Titant lo Blanch.

Gilmer Echols, Diana, Neely, Patricia \& Dusick, Diane (2018). Understanding faculty training in competency-based curriculum development in The Journal of Competency-Based Education. Vol.3, núm. 2, pp. 19. doi: $10.1002 / \mathrm{cbe} 2.1162$

Gimeno Sacristán, José (1981). Teoría de la enseñanza y desarrollo del currículo. Madrid: Anaya, S.A.

Habermas, Jürgen (2007). La lógica de las ciencias sociales. Madrid: Tecnos.

Kelly, Andrew P. \& Columbus, Rooney. (2016). Innovate and evaluate: Expanding the research based for competency-based education. Washington, DC: Center for Higher Education Reform.

Quinn, Lynn. (2019). Re-imagining Curriculum: Spaces for disruption. Stellenbosch: Sun Press.
Rabinovich, Semyon G. (2005). Measurement Errors and Uncertainties. Theory and Practice. New York, NY: Springer Science and Media, Inc. doi: $\underline{10.1007 / 0-387-29143-1}$

Salkind, Neil (2010). Encyclopedia of Research Design. Thousand Oaks. CA: SAGE Publications, Inc. doi: 10.4135/9781412961288

Samuel Michael, A., Dhunpath, R. \& Amin, N. (2016). Disrupting Higher Education Curriculum: Undoing Cognitive Damage. The Netherlands: Sense Publishers.

San Martín Gutiérrez, Sonia, Jiménez Torres, Nadia y Jerónimo SánchezBeato, Estefanía (2016). La evaluación del a lumnado universitario en el Espacio Europeo de Educación Superior. Aula Abierta. Vol. 44, Núm. 1, pp. 7-14.doi:10.1016/j.aula.2015.03.003

The National Academies of Sciences, Engineering, and Medicine (2019). Reproducibility and Replicability in Science. Wa shington, DC: National Academies Press. doi: $\underline{10.17226 / 25303}$

Wilcoxon, Frank (1945). Individual comparisons by ranking methods. Biometrics Bulletin. Vol. 1, núm. 6, pp. 80-83. doi: $\underline{10.2307 / 3001968}$ 